\title{
Free Will in Human Behavior and Physics
}

\author{
Vasil Penchev, vasildinev@gmail.com \\ Bulgarian Academy of Sciences: Institute for the Study of Societies of Knowledge: \\ dept. of Logical Systems and Models
}

\begin{abstract}
If the concept of "free will" is reduced to that of "choice" all physical world share the latter quality. Anyway the "free will" can be distinguished from the "choice": The "free will" involves implicitly a certain goal, and the choice is only the mean, by which the aim can be achieved or not by the one who determines the target. Thus, for example, an electron has always a choice but not free will unlike a human possessing both. Consequently, and paradoxically, the determinism of classical physics is more subjective and more anthropomorphic than the indeterminism of quantum mechanics for the former presupposes certain deterministic goal implicitly following the model of human freewill behavior. Quantum mechanics introduces the choice in the fundament of physical world involving a generalized case of choice, which can be called "subjectless": There is certain choice, which originates from the transition of the future into the past. Thus that kind of choice is shared of all existing and does not need any subject: It can be considered as a low of nature. There are a few theorems in quantum mechanics directly relevant to the topic: two of them are called "free will theorems" by their authors (Conway and Kochen 2006; 2009). Any quantum system either a human or an electron or whatever else has always a choice: Its behavior is not predetermined by its past. This is a physical law. It implies that a form of information, the quantum information underlies all existing for the unit of the quantity of information is an elementary choice: either a bit or a quantum bit (qubit).
\end{abstract}

Key words: axiom of choice, choice, free will, free will theorems, goal, hidden variables, quantum information, quantum mechanics

\section{The thesis of the paper}

The thesis of the paper is the following:

If the concept of "free will" is reduced to that of "choice", the physical world shares that quality. However, the physical world both in classical and in quantum physics shares the "principle of least action" though in a generalized formulation as to the former. The principle of least action can be also interpreted as a principle of most probability and thus as that of least choice and information in final analysis.

\section{Choice both in quantum mechanics and in human behavior}

The concepts of "free will" and "choice" can be juxtaposed to each other. The "free will" should be distinguished from the "choice":

The free will includes both a freely chosen goal and a relevant way to be achieved. That goal can be really achieved or not.

Mathematically defined, choice is only a relation or even a mapping between a set of alternatives and an element of this set, i.e. a given alternative (either chosen or happened). "Choice" 
does not require (but not exclude) the "goal" and "relevant way", which can be represented as a constructiveness of the choice also mathematically.

The goal suggests a constructive way to be realized. The "free will" involves a certain goal, and that way is only the mean, by which the aim can be achieved or not by the one who determines the target. Consequently, the so-defined concept of choice serves to describe the situation of choice in an abstract way, only by means of the states before and after the choice and their relation. That concept of choice is used in mathematics (set theory) and physics (quantum mechanics) being unsufficient but not irrelevant to definition of the choice of a human being. It neglects both goal and way for it to be achieved, which are specific for "free will".

Quantum mechanics allows of discussing both "free will" and choice of e.g. an electron in a sense. Thus, for example, an electron has always a choice but not free will unlike a human possessing both. However, the temporal model of choice in both cases is the same: It suggests a leap (a quantum one for the electron) into future and after that, the determination of the way from the end of the future state to the beginning of the present state. The electron determines the future state in no way, still less as "wished", which is specific for "free will", though.

One can be featured determinism in physics (or at least in quantum mechanics) as a kind of subjectivism, which seems to be rather paradoxically at first glance:

Consequently, but surprisingly, the determinism of classical physics turns out to be more subjective and anthropomorphic than the indeterminism of quantum mechanics. The former presupposes a certain deterministic goal implicitly following the model of human behavior and free will. However, on the other side, determinism can be interpreted as a particular case of the principle of least choice when any choice misses.

Choice can underlie the base of the world therefore acquiring an ontological status. The choice is usually linked to very complicated systems such as human brain or society and even often associated with consciousness. However, one can easily demonstrate that choice originates from the one of the most fundamental phenomena, the course of time. Both electron and human being share the course of time and thus both are forced to be relevant and adapted to it.

The choice is available also in the material world rather than only in the frame of human behavior:

The material world seems to be deterministic and absolutely devoid of choice. In fact, that alleged missing choice is only a particular case of the "principle of least choice", namely when no choice, i.e. the choice is zero. However one can suggest that the "principle of least choice" is predetermined by the course of time itself and thus shared by the free will of any human being as the free will should be relevant to time, too.

The concept of choice is available in quantum mechanics often implicitly. In fact, quantum mechanics introduces the choice in the fundament of physical world and even in the only way, in which it can exist: Thus quantum mechanics reveals the specific way for any quantum item to be referred to time. The time is what generates the being in final analysis though by the meditation of choice and information as the quantitative measure of the choices and their number. So, one can figuratively say that quantum mechanics "rewrites" Heidegger's "Sein und Zeit" as "Zeit und Sein". 
The present as a modus of time can be discussed as a "phase transition" from the rather undetermined future to the absolutely determined past. The metaphor of "phase transition" is an allusion to the transition between two different states of aggregation of matter in chemistry such as steam, fluid water, and ice:

Two absolute different media, that of future and that of past, collide each other. Thus, what we mean as the "present" is a phase transition between them:

All exist in the "phase transition" of the present between the uncertain future and the wellordered past. The essence and "atom" of that transition is the choice for only the choice can transform future into past.

The way of transforming future into past orders gradually the "gas phase" of the chaotic future into the semi-ordered "liquid phase" of the present and ultimately to the "solid phase" of the wellordered past:

Thus the present is forced to "choose" in order to be able to transform the coherent state of the future into the well-ordering of the past. The future is unorderable in principle, but the past is always well-ordered in time: This implies that the "phase transition" between them, i.e. the present is the only which transforms the former into the latter. Mathematically, this is the so-called "wellordering theorem" equivalent to the axiom of choice:

If the concept of choice is emancipated from the human behavior therefore acquiring an ontological meaning, it should be emancipated from any choosing in final analysis:

The concept of choice in common sense as if suggests that there is always someone who chooses. However, the axiom of choice does not suggest anybody who chooses neither also the well-ordering theorem requires anybody who orders. One might say that the time itself is what chooses or orders. However, it is still better to be introduced a special kind of subjectless choice or ordering. Choice and ordering should be thought as relations rather than actions still less as human actions.

The end point of emancipating from any choosing is the "subjectless" choice of quantum mechanics, which means "pure choice", choice by itself, or choice as a substance:

Indeed quantum mechanics involves a generalized case of choice, which can be called "subjectless": One might say that the apparatus is what chooses and thus quantum mechanics is fundamentally subjective unlike classical physics. Even more, quantum mechanics ostensibly needs a subject without whom reality might not exist. In fact, all those wrong conclusions originate from the misunderstanding of the subjectless choice involved by quantum mechanics.

The subjectless choice is what transforms future into past without the meditation of any subject of the choice. Its base is not any choosing whether a human being or an alleged "God", but mathematical laws or axioms such as the axiom of choice:

There is certain choice, which originates from the transition of the future into the past. This is the same choice introduced by quantum mechanics as the relation between the following two states:

1) The coherent state unorderable in principle for the theorems about the absence of hidden variables in quantum mechanics (Neumann 1932), (Kochen and Specker 1968);

2) The results of the measurement constituting always a well-ordered statistical ensemble. 
The kind of subjectless choice is omnipresent just for no choosing to choose: Then one can say that all choose or the whole of all is what chooses:

Thus, that kind of choice is shared of all existing and does not need any subject: The subjecteless choice of quantum mechanics can be deduced from the subjectless choice accompanying the course of time. Consequently, the time is what can relate to each other and even unify the subjectless choice in quantum mechanics and the freewill choice of a human being: Indeed any item in the universe should be adequate to the course of time in order to be able to exist.

Choice can be also considered as a universal law of nature as anything cannot help but choose for anything is in the present above defined as the medium of choice:

Choice can be considered even as the most universal law of nature. That law originates from time: So, choice should be a universal law not only of nature but not less, of consciousness, too. A human being unlike an electron possesses consciousness, but both share choice for both are relate to time. So, choice is the universal law of nature and consciousness.

\section{The "free will theorems"}

There are a few theorems in quantum mechanics directly relevant to the topic: two among them are called "free will theorems" by their authors (Conway and Kochen 2006; 2009). A mathematically absolutely rigorous way for these theorems to be proved is demonstrated by the two scientists in their paper. Furthermore, their conclusions can be grounded philosophically on the base of quantum holism, i.e. by means of the quantum and holistic unity of the quantum entity and experimenter. Choice is what really unifies all and thus can be interpreted as a kind of the solidarity of anything as being in time:

If the experimenter possesses "free will", the holistic unity implies the same for the electron. However, one can found the unity of the electron and human being in another way: Both are forced to be "solidary" in front of the time, of the future. So, both share choice for that solidarity. Even more, they should be a coherent whole in their common future and only the choice is what can isolate them from each other in the present.

A few quotations from the "free will" papers are sufficiently revealing:

Do we really have free will, or, as a few determined folk maintain, is it all an illusion? We don't know, but will prove in this paper that if indeed there exist any experimenters with a modicum of free will, then elementary particles must have their own share of this valuable commodity. (Conway and Kochen 2006, 1441)

This quotation can be seen as a form of "quantum holism".

The conclusion of the second paper is: "The import of the free will theorem is that it is not only current quantum theory, but the world itself that is non-deterministic, so that no future theory can return us to a clockwork universe" (Conway and Kochen 2009, 232). If the world itself is "nondeterministic", and the universe is not "clockwork", this means that the choice can be put in their base. 
The law of least choice or information (which can be zero as a borderline case) is what creates the illusion of a "clockwork" and deterministic universe without any choice.

The picture of a universe of free will rather than "clockwork" can explain the absence of "hidden variables" in quantum mechanics. Those parameters, which are missing, would redefine the universe to a "clock" working absolutely deterministically. However after that is not the case, those hidden variable have to miss in nature:

The "free-will theorems" can be considered as a continuation of the so-called theorems about the absence of "hidden variables" in quantum mechanics (Neumann 1932, 167-173), (Kochen and Specker 1968). They refute the hypothesis (of Einstein and others) about hidden but unknown variables after the reveal of which, ostensibly, quantum mechanics would become "complete". The quoted theorems deduce this rigorously from the mathematical formalism used by quantum mechanics.

Choice and hidden variables naturally contradict to each other:

In fact, those "hidden variables" are necessary for there be no choice. In other words, if the choice is not only admitted but even put in the base of the universe, any hidden variables are refuted rather than only redundant. In a sense, the "free will theorems" should imply the earlier "no hidden variables" theorems. Consequently, the "free will" theorems are stronger than the "no hidden variables" theorems. However, if quantum information (the quantity of choices) is that hidden variable, the formers are only a different result consistent both with "hidden variables" and with "no hidden variables".

Furthermore, quantum measurement can be considered as a kind of ordering. Indeed any measurement can be interpreted as a choice of a value among the set of all possible values. The realized measurement has ordered the chosen value in time thus continuing the process of wellordering of all possible values. The "no hidden variables" theorems imply no well-ordering in any coherent state in any quantum system before measurement. However, the same state is transformed into a well-ordered (e.g. by the parameter of time) statistical ensemble after measurement. So, the "no hidden variables" theorems imply in turn some choice of the measured quantum entity and thus the free will theorems.

Consequently, the "no hidden variables" and "free will" theorems should be equivalent in a sense.

All this addresses a certain philosophical conclusion:

The eventual equivalence of the "no hidden variables" and "free will theorems" implies the following: Quantum information as an alleged "hidden variable", being the quantity of choices, should be equivalent to all the rest in the physical world. Indeed the above equivalence is logically possible then and only then when: the world (in the case of "no hidden variable") is exactly the same as (or exactly equal to) quantum information (in the case of the only hidden variable of quantum information).

The above conclusion can be even continued to a philosophical "speculation":

If quantum information is the universal substance of the world being equivalent to it and quantum information is the quantity of choice, then: Choice grounds the universe. Even more, the choice itself can be founded by the course of time. So, time can be put in the base of all being. 


\section{Interpretations}

The results of any measurement including quantum measurement are always well-ordered:

Indeed the measured results by any quantum measurement are always well-ordered (e.g. by the parameter of time). If they refer to one and the same coherent state, they represent it as a statistical ensemble in final analysis. As that statistical ensemble can be obtained only by well-ordering of an unorderable state as the coherent one is, the meditation of transfinite ordinal numbers is necessary. Thus, the link of the state before and after measurement implies the well-ordering theorem equivalent to the axiom of choice.

Furthermore, the state before measurement as being unorderable in principle involves some infinite set implicitly for any finite set can be always ordered somehow. Therefor the corresponding ordinal number has to be transfinite for no finite ordinal number can corresponds to an infinite set after the well-ordering of it.

There are two definitions of "ordinal number":

- In Neumann (1923);

- In Cantor (1893) - Russell.

Both definitions are applicable to the equivalent of a coherent quantum state after measurement. However, they generate different though perhaps equivalent concepts as the measure of quantum information:

- In Neumann: a binary transfinite number;

- In Cantor - Russell: a statistical ensemble.

The choice being omnipresent or "subjectless" therefore is shared by all in the universe:

So, any quantum system whether a human or an electron, or whatever else has always some choice: Thus the choice can be considered as the omnipresent resource for general use in the universe shared by any item including an electron and a human being. The source of this resource is the time. Time is the only which grants the choice as a "gift" for all in the universe.

The choice is what generates the past in a sense since the latter is always a series well-ordered in time. That series needs the choice in order to be able to be created from the "chaotic matter" of the future.

The behavior of any item in the universe is not predetermined by its past in general. The determinism is only a particular, borderline, and idealized case according to the "law of least choice" when the choice and thus information is zero. In fact, the choice and information in a trajectory can be zero when and only when the segment of the trajectory in question is thoroughly in the past. Consequently, determinism is the idealization of the case when a motion is almost in the past for the big mass of the moving: That is the case in classical physics (No motion can be actually and absolutely in the past for there is no motion then).

However, the past being absolutely ordered (or mathematically said, well-ordered), "crystalized" is also unchangeable, "effortless", and even "dead".

Indeterminism is a physical law. Even more, indeterminism is a universal law of the being which is in the time. Motion whether mechanical or any physical, or whatever else means indeterminism, choice and thus information. Motion cannot happen if it is really and absolutely 
predetermined. The past is effortless, "dead": No motion can occur in the past. The past is always well-ordered and thus unchangeable.

There is a direct link between choice and information. Indeed information can be generally defined as a relation between two orderings or as the way the one of them to be transformed into the other by a series of elementary steps. Any that step is an elementary or minimal choice such as a bit or a qubit. Thus information and quantum information as quantities are the number of bits or qubits, which distinguish the orderings from each other. If the one ordering is some zero benchmark, e.g. the quantity of information of a coherent state as an absolutely unorderable "chaotic gas phase", the quantity of information of the other ordering depends only on itself:

However, quantum information underlies not only any physical motion but also any physical item ("particle"). This implies that a form of information, the quantum information underlies all existing. The unit of the quantity of information is an elementary choice: either a bit or a quantum bit (qubit). The boundary between "bit" and "qubit" is infinity: A bit means a choice between two alternatives (i.e. a finite number) while a qubit means a choice of an element among an infinite set of alternatives.

If the choice is what unifies all in the world, this implies the common base of quantum information:

Thus both experimenter and electron share a common fundament of choice and quantum information. That common choice and thus quantum information originate from their joint existing (co-existing) in the present. They cannot help but share some minimal nonzero quantity of choice being coexisting. The phenomenon of entanglement can be defined as shared quantum information originating from the shared choice.

The course of time and that of entanglement are opposite in a sense if the direction of time is accepted to be according to the usual "arrow" from the determined past to the more undetermined future. Indeed the entanglement "moves back": from the coherent state, which is inherent to the future, through the partly and decreasingly entangled state (of the present) to the absolutely unentangled state, which is equivalent to the well-ordered state (of the past).

What features the choice of an electron from the analogical choice of a human being is the goal of the latter. They can be distinguished from each other in a mathematical sense: The choice of an electron is fundamentally random being guaranteed by the axiom of choice. It does not address any "goal" or "state" wished by the electron. Indeed this would be quite ridiculous. A human being's choice can be constructive, directed to some wishful future state and include a relevant pathway or method to be achieved. All this is meant in the term "goal", which is an additional option featuring just the human choice and included in "free will", which includes also "choice".

Anyway a human (e.g. an experimenter) can predetermine a goal for achieving while an electron cannot do it in principle. However, of course, the experimenter cannot predetermine the "choice of the electron" as experimenter's goal and choice. This would mean that the experimenter might deprive the electron of its legitimate choice and therefore, violate a fundamental law of nature: Its violation is impossible and even absurd.

The goal is what distinguishes human free will and the choice of an electron: It is inherent to the former, but absolutely absent in the latter. 
Consequently, what distinguishes the human free will from the free choice of an electron is the goal: However any human being's free will and goals should be limited: Not only by the other human beings' free will and goals, but furthermore by the freedom of choice of anything in the universe. The free will theorems means: The experimenter's behavior obeys "categorical imperative" as the principle of the universe rather than of human society only.

The target of a human being and the random choice of an electron can be opposite to each other even in the "axis" determinism - indeterminism: The former is on the former pole of that axis, the latter correspondingly on the latter one. Rather paradoxically, the determinism of classical physics should be equated rather to the human target than to the choice shared by all in the universe. So, classical physics turns out to be more anthropomorphic than quantum mechanics too often and groundlessly alleged to be "subjective" unlike the ostensibly "objective" classical physics.

Free will supposes a target while the choice of an electron is always random. The electron's choice is guaranteed mathematically by the axiom of choice. The electron does not dispose of any constructive way such as plans, targets to achieve its choice. Nevertheless its choice is guaranteed as a law of nature not less than that of a human being supplied by consciousness and thus by constructive methods to all permissible goals. Permissible goals are what do not contradict the "categorical imperative" of the universe.

The subjectivity of classical physics can be seen so:

Rather unexpectedly, classical physics turns out to be more anthropomorphic than quantum mechanics. Quantum mechanics was alleged to be dependent on an experimenter or even on an observer ostensibly deciding for the measured result what to be. This is not true, though. In fact the determinism of classical physical deprives anything in the universe except the human being of the freedom of choice: a form of human chauvinism, subjectivism and anthropocentrism. This is wrong, too.

The omnipresent choice implies that indeterminism unlike determinism is the universal law of nature:

In fact, free choice is shared by all existing while determinism features only beings having free will as a human but only as a self-deception. In fact, the human free will is limited by "categorical imperative" guaranteeing the free choice of anything in the universe. Consequently, the human free will is both only a form of the omnipresent choice and restricted by the same omnipresence of choice.

The anthropomorphism of classical physics originates from the model of human behavior striving to certainty, determinability, and maximal control. Only so, the human goals would be able to be achieved:

Classical physics remaining still anthropomorphic describes nature deterministically and similarly to human behavior. Thus, it conserves the choice ostensibly as a resource only for the human beings: This is self-deception, which consists in the fundamental misunderstanding even of what a human being is and of how the human being differs from the rest in the universe. 


\section{Conclusions:}

The main conclusion of the paper is:

The free will theorems in quantum mechanics can be interpreted philosophically. That interpretation leads to the free-choice ontology of quantum mechanics. Choice and quantum information as the quantity of choices is the base of the universe. Both electron (as an example for any quantum item) and experimenter share the common resource of choice. "Categorical imperative" as a law of universe guarantees the "right of choice" of any item in the universe either a human being or an electron, or anything else in the universe.

A few additional conclusions can be abstracted:

The human free well includes some goal as well as a constructive way whether true or false for it to be achieved. The choice guaranteed for any item in the universe (thus for any human being, too) is not constructive in general, and random in principle. The choice and its quantity of quantum information originate from the course of time and thus are shared by anything. The choice is the only way for the unorderable in principle coherent state of future to be transformed into the well-ordered past by the meditation of the present and choice. 


\section{References:}

Cantor, Georg (1897) "Beitrage zur Begrundung der transfiniten Mengenlehre (Zweiter Artikel)." Mathematische Annalen 49 (2): 207-246.

Conway, John, and Simon Kochen (2006) "The Free Will Theorem." Foundations of Physics 36 (10): $1441-1473$.

Conway, John, and Simon Kochen (2009) "The Strong Free Will Theorem." Notices of the AMS 56 (2): 226-232.

Kochen, Simon, and Ernst Specker. (1968) "The problem of hidden variables in quantum mechanics." Journal of Mathematics and Mechanics 17 (1): 59-87.

Neumann, John von (192). "Zur Einführung der trasfiniten Zahlen." Acta litterarum ac scientiarum Ragiae Universitatis Hungaricae Francisco-Josephinae, Sectio scientiarum mathematicarum 1 (4): 199-208.

Neumann, John von. 1932. Mathematische Grundlagen der Quantenmechanik, Berlin, Springer

Whitehead, Alfred North, and Bertrand Russell (any edition) Principia Mathematica, Vol. 2 (*153), Vol. $3(* 251)$. 